\title{
Experimental Study on Vibration Transmissibility Properties of Honeycomb Paperboard
}

\author{
Quanfu Gao ${ }^{1}$, Dapeng Zhu ${ }^{*}, 2$ and Xingxiao Cao ${ }^{1}$ \\ ${ }^{1}$ School of Mechanical Engineering, Lanzhou Jiaotong University, Lanzhou 730070, P.R. China \\ ${ }^{2}$ School of Traffic and Transportation, Lanzhou Jiaotong University, Lanzhou, 730070, P.R. China
}

\begin{abstract}
The vibration transmissibility properties of a honeycomb paperboard are investigated by experiments. The analysis of the steady-state response of mass loaded honeycomb paperboard system to harmonic base excitation is presented in this paper. An experiment system is formulated and the vibration transmissibility data are obtained under different base excitation amplitude conditions. According to the transmissibility experiments data, we can assume that both the stiffness and damping properties are nonlinear. The hysteresis loops of the honeycomb paperboard-mass system are experimentally obtained, and are used to investigate the stiffness and damping properties of the system. The honeycomb paperboard's uni-directional vibration transmissibility behavior is modeled by using nonlinear stiffness, the combination of velocity proportional damping and quadratic type nonlinear damping components. The parameters in the model are identified by use of one-term harmonic balance method and the parameters under different load condition are presented. The model and the parameters in this paper can be used to simulate the transmissibility-frequency curves accurately. The model can be used for understanding the vibration transmissibility behaviors of the honeycomb paperboard, and the simulated transmissibility-frequency curves can be used for the packaging design.
\end{abstract}

Keywords: Honeycomb paperboard, nonlinearity, parameters identification, vibration transmissibility properties modeling.

\section{INTRODUCTION}

Honeycomb paperboard is like a sandwich panel which consists of three layers: the upper and lower liners, while the honeycomb core lies between the two layers. These parts are composed of reusable paper. Due to its special structure, it has many advantages over other materials, like its high strength-to-weight ratio, high stiffness-to-weight ratio, light weight, and the ease of processing. In addition, it is recyclable, reusable and biodegradable. Because of these obvious benefits, honeycomb paperboard is being used by many industries. Recently, keeping the environment protection concerns in view and the need for reducing the plastic waste, people have started using honeycomb paperboard in the protective packaging and packing as an alternative of foam. The consideration of the honeycomb paperboard as an important cushion material has encouraged a close scrutiny of its properties.

Guo and Zhang have investigated the cushion properties and vibration transmissibility properties of honeycomb paperboard consisting of varied thickness in a series of experiments. The experiment results are fitted by polynomials. These results have provided the basic data for protective packaging designing $[1,2]$. Wang has investigated the cushioning properties of honeycomb paperboard by experimental analysis [3] in which the structure of honeycomb paperboard, relative density of paper honeycomb cores, liners and layouts of the cushioning properties have been studied. The impact behavior and energy absorption properties of honeycomb paperboard have been presented by Wang [4]. The experimental results have shown that the increase in the relative density of paper honeycomb cores can efficiently improve the dynamic cushioning properties as well as the thickness of paper honeycomb cores has a fluctuant effect on the cushion properties. Hidetoshi Kobayashi et al. [5] studied the effect of loading rate on the strength and absorption energy of the cores by means of the quasi-static and dynamic compression experiments. The critical buckling load of honeycomb paperboard under outof-plane pressure was investigated by analyzing the structure and the collapse mechanism [6]. The models and the calculation method in the paper can be used to predict the static critic buckling load. Zhu $[7,8]$ modeled honeycomb paperboard as a linear material with viscoelastic property, the relaxation kernel was expressed as the sum of complex exponentials, a parameter identification procedure was formulated using the free response data of honeycomb paperboard-mass system based on the modified Prony method, the identified parameters can be used to predict the forced and free response of honeycomb paperboard-mass system.

The research above provided the basic knowledge of honeycomb paperboard properties, but there are few references about the vibration transmissibility property of honeycomb paperboard. The vibration transmissibility $T_{r}$ is defined as

$$
T_{r}=|\ddot{x}| /|\ddot{y}|
$$


where $T_{r}$ is the acceleration transmissibility, $\ddot{x}$ and $\ddot{y}$ are the acceleration output and input of the packaging system respectively, $\mid \cdot$ denotes the amplitude of the vibration data. The acceleration transmissibility is usually the function of vibration frequency $f$, i.e. the vibration transmissibility properties of the honeycomb paperboard can be expressed by the $T_{r}-f$ curves, and the $T_{r}-f$ curves are important for the packaging design. In this paper we have presented the experimental and analytical investigations for examining the properties of honeycomb paperboard transmissibility in unidirectional deformation. Most importantly we have investigated the effects of excitation amplitude on the transmissibility properties. A phenomenological modeling of the honeycomb paperboard mechanical properties is developed and used in the governing equation of motion of the honeycomb paperboard isolator. The differential equation of motion includes the stiffness nonlinearity and the damping nonlinearity. The parameters in the equation are identified by use of harmonic balance method. This model can be used to predict the isolator dynamic characteristics and its transmissibility for the excitation vibration with different amplitude.

\section{EXPERIMENTAL INVESTATION}

Because of the greater strength of honeycomb paperboard, the amount of compression is very small under some load, thus, in many cases, researchers have regarded honeycomb paperboard as a linear material $[1,7,8]$. Nevertheless, nonlinear characteristics in compression deformation exist in the mechanical properties such as stiffness and damping. Moreover, even when the excitation amplitude is small the response amplitude may often be large enough that nonlinearities cannot be ignored, especially at the resonance frequency.

To learn the dynamic properties of the mass loaded cushion material system, researchers usually use random noise or a continuous frequency sweep as the input excitation. There are particular deficiencies with both methods when applied to nonlinear system identification. The use of random input and traditional frequency response estimation procedures using cross and power spectral densities [9] produces the best linear approximation to the system corresponding to this particular input, and therefore will not exhibit a nonlinear shape. Alternatively, the response to a continuous frequency sweep is often used for generating the system frequency response. Although it does not inherently assume system linearity, there are problems associated with this method. In particular, the sweep rate can have large effects on the measured response, this is true even for linear system. Faster sweep smooth out many of the features of the system. A very slow sweep rate will alleviate this problem to a certain degree, but unless careful precautions are taken, the measurement of fine detail of the steady state response is not assured, thus making identification more difficult.

As described above, the measurement technique used in this paper consists of discrete frequency stepping, where the system is vibrated at a single frequency until the steady state condition is achieved. Then the amplitude of the response and the phase change between the acceleration response and the specimen deformation at the excitation frequency are measured. The frequency is then increased to the next value, and the process repeated to generate $T_{r}-f$ curves.

\subsection{Experimental Set-Up}

The vibration transmissibility properties of honeycomb paperboard are investigated by the experiment system shown in Fig. (1). In this experiment system, a single-degree-offreedom system is fabricated by inserting the honeycomb paperboard specimen between a rigid mass and a harmonically moving base. The inertia of the rigid mass is set to be $10 \mathrm{~kg}$. The specimens are cut to be $250 \times 250 \mathrm{~mm}^{2}$, the thickness of specimens is $40 \mathrm{~mm}$, the basic weight of the liners is $300 \mathrm{~g} / \mathrm{m}^{2}$, the honeycomb core is made of reusable paper with basic weight of $150 \mathrm{~g} / \mathrm{m}^{2}$. The honeycomb paperboard specimen is glued to thin metal plates which are in turn bolted to the moving base and the rigid mass to prevent them from losing contact with each other during vibration. Before the tests, all the test specimens are preprocessed for 24 hours at temperature $21^{\circ} \mathrm{C}$, and relatively humidity $44 \%$.

Two accelerometers are used to record the system acceleration input and output, a compressible displacement transducer is fixed between the rigid mass and shaker table to monitor the displacement of the honeycomb paperboard specimen at each sampling instant. The high frequency noise in all the signals is cut off by using a low-pass filter. The cut off frequency of the filter was set at $3 \mathrm{kHz}$. The acceleration signals, along with displacement signal, are digitized by a YE6230B dynamic data acquisition equipment (16 bit) and transferred to a PC. All the data record process in this work is under the control of the YE7600 software package. We set the sampling frequency to be 50000 samples per second to calculate the phase change $\ddot{o}$ between system acceleration response and specimen deformation accurately.

\section{VIBRATION TRANSMISSIBILITY MEASUREMENTS}

The rigid mass and honeycomb paperboard specimen formulate a mass-material system in Fig. (1), this system is used to simulate a package. As with any nonlinear system, the control of input frequency and amplitude is of critical importance to the measured results. Before the measurements, the system is excited at a constant acceleration level over various frequencies for 1 hour, this amount of excitation allowed the honeycomb paperboard specimen to come to its ultimate equilibrium compression level. Then the mass-material system is axially excited with a constant sinusoidal acceleration level by the shaker table at frequency $f$. The acceleration response $\ddot{x}$ and the excitation acceleration $\ddot{y}$ of the system are measured by accelerometers, the vibration transmissibility of the system $T_{r}$ at this frequency is obtained by use of Equation (1), the phase change ö between $\ddot{x}$ and $z$ is also calculated and recorded. The input frequency is then slowly increased to the next value and repeat the measurement process. In regions sufficiently far away from resonance, the step in frequency is $5 \mathrm{~Hz}$. However, in regions near the resonance, the frequency step is reduced to $0.5 \mathrm{~Hz}$ in order to accurately capture the data at the resonance frequency. After this measurement process, we can obtain a steady state $T_{r}-f$ curve to represent 


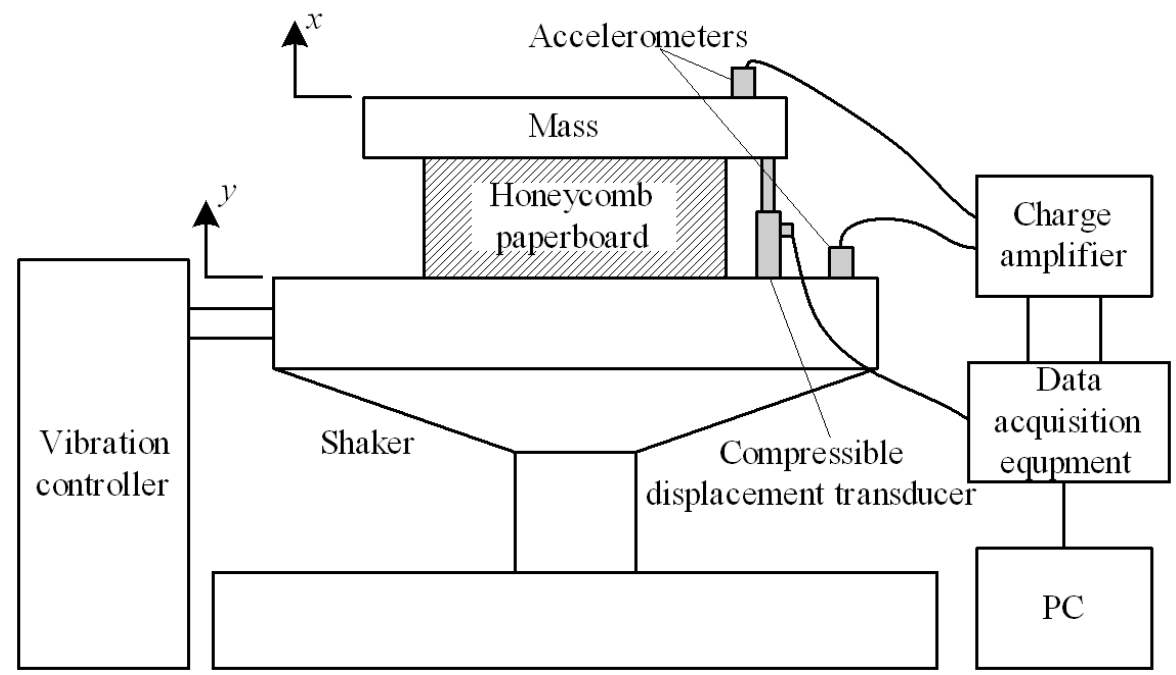

Fig. (1). Schematic of setup for honeycomb paperboard testing.

the vibration transmissibility properties of honeycomb paperboard.

As nonlinear material, the vibration transmissibility properties of honeycomb paperboard are influenced by the excitation amplitude. Fig. (2) shows the measured $T_{r}-f$ curves for different values of excitation acceleration amplitude $|\ddot{y}|$.

In Fig. (2), the transmissibility measurements show that the resonance peaks are shifted to the left from $131.5 \mathrm{~Hz}$ to 120 $\mathrm{Hz}$, and the peak resonance magnitudes are reduced from 5.17 to 3.44 as the excitation magnitude increases. This indicate that as the excitation amplitude increases, the stiffness decreases and damping increases.

\section{VIBRATION TRANSMISSIBILITY PROPERTY MODELING}

From Fig. (2), we can assume that both the stiffness property and damping property are nonlinear functions of honeycomb paperboard deformation $z(z=x-y)$. To study the dependence of dynamic characteristics on the deformation amplitude, tests are conducted at a particular frequency with varying excitation amplitudes. Fig. (3) shows the hysteresis loops for different deformation amplitudes at the frequency of $120 \mathrm{~Hz}$. When the shaker moves, the inertia force of the mass is the external dynamic force acting on the honeycomb paperboard specimen. This inertia force $F$ is obtained by measuring the acceleration of the rigid mass $\ddot{x}$, i.e. $F=m \ddot{x}$. The deformation of the specimen $z$ is obtained by use of the compressible displacement transducer.

It is clear from Fig. (3) that, the dynamic stiffness of the honeycomb paperboard specimen is amplitude-dependent and has a softening type characteristic. The major axis of the hysteresis loop can be used to represent the stiffness force, and it can be expressed as the polynomials of specimen deformation. It can be seen that the major axis is symmetric function about $z$. Thus, a good starting point in the modeling process for the elastic properties of the honeycomb paperboard specimen would be to approximate the dynamic stiffness force by a polynomial of the form

$F_{k}=k z+k_{3} z^{3}+k_{5} z^{5}$ where $F_{k}$ is the elastic force in the honeycomb paperboard specimen and $z$ is the uniaxial compression in the material around a compression level.

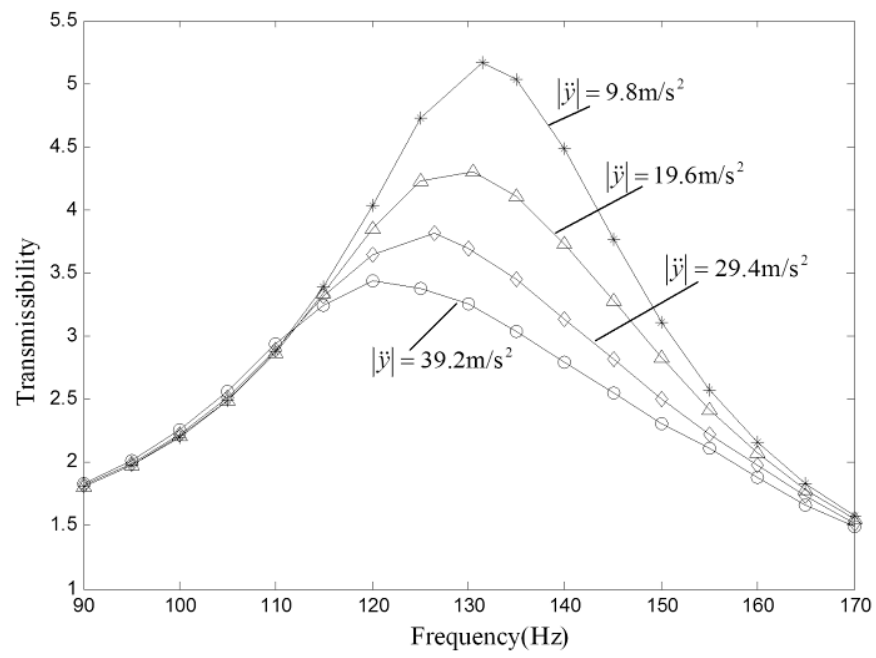

Fig. (2). Measured $T_{r}-f$ curves of honeycomb paperboard for different excitation amplitudes.

The area enclosed by the hysteresis loops in Fig. (3) is equivalent to the damping energy loss $\ddot{A} E$, and $\ddot{A} E$ can be estimated by the use of trapezium rule. Fig. (4) shows the variation of the damping energy loss $\ddot{A} E$ with the deformation amplitude $|z|$ at the frequency of $120 \mathrm{~Hz}$. Fig. (4) suggests that $\ddot{A} E$ is approximately proportional to $|z|^{2.4}$. It is known that for the linear viscous damping, $\ddot{\mathrm{A}} E$ varies linearly with $|z|^{2}$, for the quadratic type nonlinear damping, $\ddot{\mathrm{A}} E$ varies linearly with $|z|^{3}$. Thus, we can assume the damping force $F_{d}$ in the honeycomb paperboard specimen as the combination of linear damping force and quadratic nonlinear damping, i.e.

$F_{d}=c \dot{z}+c_{2} \dot{z}|\dot{z}|$

where $\dot{z}$ is the compression velocity of the honeycomb paperboard specimen, $c$ and $c_{2}$ are the linear and quadratic type nonlinear damping coefficients respectively. 


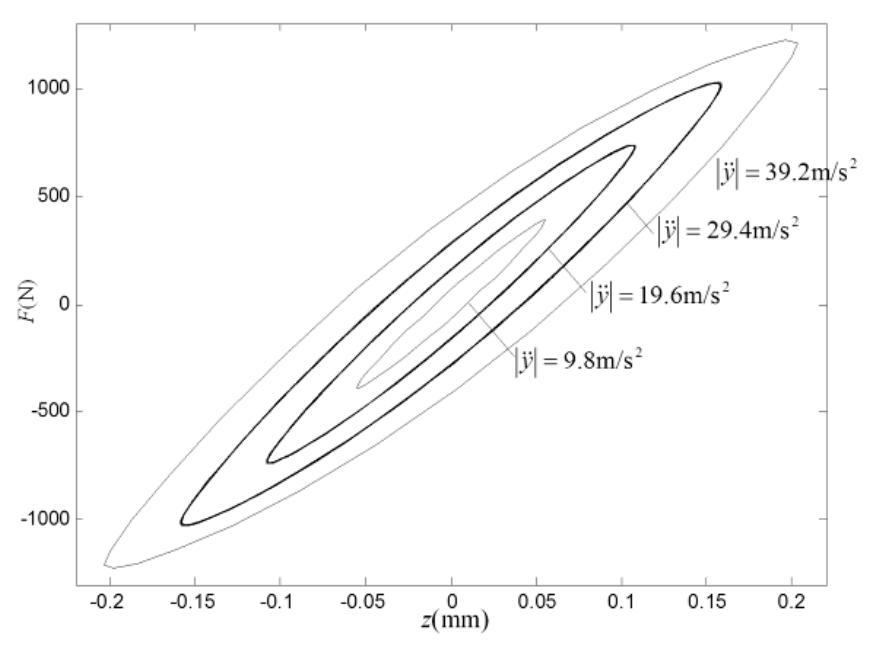

Fig. (3). Hysteresis loops at $120 \mathrm{~Hz}$ with different excitation amplitude.

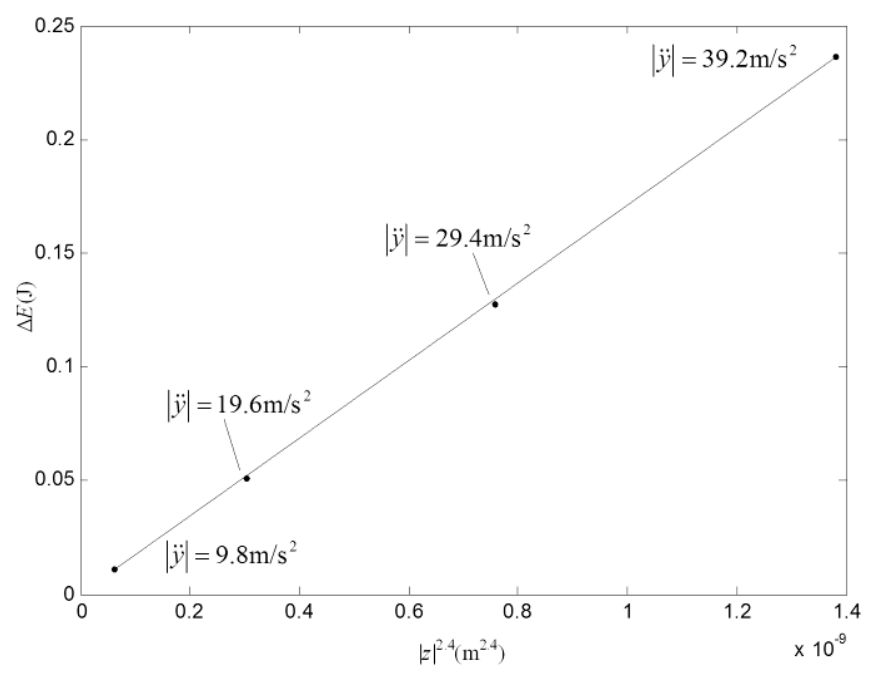

Fig. (4). Variation of damping energy loss with the deformation amplitude at $120 \mathrm{~Hz}$.

From equation (2) and (3), the restoring force in the honeycomb paperboard is assumed to possess the following form:

$$
F(t)=c \dot{z}+c_{2} \dot{z}|\dot{z}|+k z+k_{3} z^{3}+k_{5} z^{5}
$$

Thus, for the base excited material-mass system shown in Fig. (1), the deformation of the specimen is governed by the following differential equation

$m \ddot{z}+c \dot{z}+c_{2} \dot{z}|\dot{z}|+k z+k_{3} z^{3}+k_{5} z^{5}=-m \ddot{y}$

where $m$ is the isolated mass, $y=|y| \cos u$ t is the base displacement.

\section{PARAMETERS IDENTIFICATION}

Equation (5), governing the behavior of the singledegree-of-freedom nonlinear system, is a nonlinear differential equation. Both the damping and stiffness properties of honeycomb paperboard are nonlinear. There are no known closed form solutions of Equation (5). However, for a harmonic input, approximate periodic solutions can be constructed by using the method of harmonic balance
$[10,11]$. In this method, it is assumed that the system has a periodic solution of appropriate period. The Fourier series coefficients for the approximate periodic solution are obtained by the usual method of substituting the solution into the differential equation and equating coefficients of like terms.

In Equation (5), we can assume the base excited force as

$$
f(t)=-m \ddot{y}=G \sin (\omega t)
$$
[12]

Then the solution to Equation (5) can be assumed to be

$$
z(t)=\sum A_{k} \sin k(\omega t+\alpha)
$$

Neglecting the super- and sub-harmonics, one can obtain

$$
z=A \sin (\grave{u} t+\ddot{o})
$$

In Equation (5), the equivalent viscous damping of the quadratic type nonlinear damping is [13]

$c_{e}=\frac{8}{3 \pi} c_{2} \omega A$

From Equation (5), (6) and (7), the vibration transmissibility property model of the honeycomb paperboard can be written equivalently as

$$
m \ddot{z}+\left(c+\frac{8}{3 \pi} c_{2} \omega A\right) \dot{z}+k z+k_{3} z^{3}+k_{5} z^{5}=G \sin (\omega t)
$$

When Equation (7) is substituted into Equation (8), the $\sin 3(\grave{u} t+\ddot{o})$ and $\sin 5(\mathrm{u} t+\ddot{o})$ terms are neglected, and the coefficients of $\sin (\grave{u} t+\ddot{o})$ and $\cos (\grave{u} t+\ddot{o})$ are equated, it gives

$$
\begin{aligned}
& A k+\frac{3}{4} A^{3} k_{3}+\frac{5}{8} A^{5} k_{5}-m A \omega^{2}-G \cos \phi=0 \\
& A \omega c+\frac{8}{3 \pi} A^{2} \omega^{2} c_{2}+G \sin \phi=0
\end{aligned}
$$

Given the system parameters, the amplitude of the response $A$ and the phase change $\ddot{o}$ can be determined by simultaneously solving (9a) and (9b). In this paper, these equations are used for system identification.

In this section, the estimation of system parameters: $c, c_{2}$, $k, k_{3}, k_{5}$, from steady-state periodic response data $A$ and $\ddot{o}$, at various forcing frequencies $\grave{u}$ and amplitudes $G$, is addressed. The mass $\mathrm{m}$ is known apriori. Let Equations (9a) and $(9 \mathrm{~b})$ be represented symbolically as $f_{1}\left(G_{i}, \grave{u}_{i j}, A_{i j}, \ddot{o}_{i j}\right)$ and $f_{2}\left(G_{i}, \grave{u}_{i j}, A_{i j}, \ddot{o}_{i j}\right)$. Where $G_{i}(i=1,2,3,4)$ is the amplitude of the base excited force and $G_{1}=98 \mathrm{~N}, G_{2}=196 \mathrm{~N}, G_{3}=294 \mathrm{~N}$, $G_{4}=392 \mathrm{~N}, \quad \grave{u}_{i j}(j=1,2, \ldots, M)$ are the $M$ input angular frequencies $(\grave{u}=2 ð f)$ at which the response amplitude $A_{i j}$ and the phase changes $\ddot{o}_{i j}$ are measured and recorded. The cost function minimized for the estimation of the parameters is

$$
\varepsilon=\sum_{i=1}^{4}\left\{\sum_{j}^{N}\left|f_{1}\left(G_{i}, \omega_{i j}, A_{i j}, \phi_{i j}\right)\right|^{2}+\left|f_{2}\left(G_{i}, \omega_{i j}, A_{i j}, \phi_{i j}\right)\right|^{2}\right\}
$$

In Equation (10), the cost function $a$ is evaluated for a combination of four input altitudes. The minimization of $a$ in Equation (10) leads to a set of equations that are linear functions of system parameters $\left(c, c_{2}, k, k_{3}, k_{5}\right)$. The 
parameters identification process can be concluded as follows:

Step 1. The mass loaded honeycomb paperboard system is excited by the shaker at frequency $\grave{u}_{i j}$, the amplitude of the excitation force is $\mathrm{Gi}$, where $G=m|\ddot{y}|$. The system is base excited for 30 minutes to reach its steady state, and then the compression of the honeycomb paperboard specimen $z(t)$, the excitation and response acceleration $\ddot{x}$ and $\ddot{y}$ are recorded. Record the amplitude $A_{i j}$ of $z(t), A_{i j}=|z(t)|$, and estimate the phase change $\grave{u}_{i j}$ between $z(t)$ and $f(t)$.

Step 2. Change the excitation frequency $\grave{u}_{i j}(j=1,2, \ldots$, $M)$, and repeat step 1 .

Step 3. Change the amplitude of excitation force $G_{i}(i=1,2,3,4)$, repeat step 1 and step 2 , record $A_{i j}$ and $\ddot{o}_{i j}$ at different excitation frequencies $\grave{u}_{i j}$ and different excitation force amplitudes $G_{i}$.

Step 4. Minimize $\stackrel{a}{a}$ in Equation (9) with respect to the remaining parameters. The parameters $c, c_{2}, k, k_{3}, k_{5}$ can be identified by least square methods, and are shown in Table $\mathbf{1 .}$

Since at different load condition, the honeycomb paperboard has different stiffness and damping properties [8]. To investigate the vibration transmissibility property of honeycomb paperboard under different load conditions, experiments are carried out and the system parameters are identified under other load conditions $(m=5 \mathrm{~kg}, m=15 \mathrm{~kg})$. The identified parameters are also presented in Table $\mathbf{1}$.

Table 1. Identified parameters in the model.

\begin{tabular}{|c|c|c|c|c|c|}
\hline $\mathbf{m}$ & $\begin{array}{c}\mathbf{k} \times \mathbf{1 0}^{6} \\
\mathbf{( N / m )}\end{array}$ & $\begin{array}{c}\mathbf{k}_{3} \times \mathbf{1 0}^{13} \\
\left(\mathbf{N} / \mathbf{m}^{\mathbf{3}}\right)\end{array}$ & $\begin{array}{c}\mathbf{k}_{5} \times \mathbf{1 0}^{\mathbf{1 9}} \\
\left(\mathbf{N} / \mathbf{m}^{\mathbf{5}}\right)\end{array}$ & $\begin{array}{c}\mathbf{c} \times \mathbf{1 0}^{3} \\
(\mathbf{N s} / \mathbf{m})\end{array}$ & $\begin{array}{c}\mathbf{c}_{2} \times \mathbf{1 0}^{\mathbf{3}} \\
\left(\mathbf{N s}^{\mathbf{2}} / \mathbf{m}^{\mathbf{2}}\right)\end{array}$ \\
\hline \hline $5(\mathrm{~kg})$ & 7.121 & -1.66 & 4.866 & 0.699 & 4.758 \\
\hline $10(\mathrm{~kg})$ & 7.084 & -2.678 & -3.35 & 1.18 & 9.453 \\
\hline $15(\mathrm{~kg})$ & 7.207 & -6.105 & 38.386 & 1.575 & 13.857 \\
\hline
\end{tabular}

The identified parameters in Table $\mathbf{1}$ can be used to simulate the vibration transmissibility curves of honeycomb paperboard under different load conditions, which provide the design and theoretical basis for the packaging designs. Substituting the parameters in Table 1 into Equation (5), the displacement response of the honeycomb paperboard specimen $z(t)$ excited by the harmonic excitation force $f(t)=G \sin (\grave{u} t)$ can be simulated by the Runge-Kutta method. Because the displacement of the base is $y=-G /\left(m \dot{u}^{2}\right) \sin (\grave{u} t)$, then the displacement response of the mass $x(t)$ can be estimated by

$$
x=z+y=z-G /\left(m \grave{u}^{2}\right) \sin (\grave{u} t)
$$

Neglecting the super- and sub-harmonics in the displacement response, we can estimate the vibration transmissibility by

$$
T_{r}=|\ddot{x}| /|\ddot{y}| \approx|x| /|y|
$$

Calculate $T_{r}$ by Equation (11) and (12) at different excitation frequency, we can simulate the vibration transmissibility-frequency $\left(T_{r}-f\right)$ curves. In Fig. (5), the simulated $T_{r}-f$ curves are presented $(m=10 \mathrm{~kg})$, the measured transmissibility data are also shown in the figure for comparison.

From Fig. (5), we can conclude that the simulated $T_{r}-f$ curves have good agreement with the experimental data, this means Equation (4) and the parameters presented in Table 1 can be used to model the vibration transmissibility of honeycomb paperboard under different excitation conditions and different load conditions. The model presented in this paper and the simulated $T_{r}-f$ curves can be used for the packaging designs.

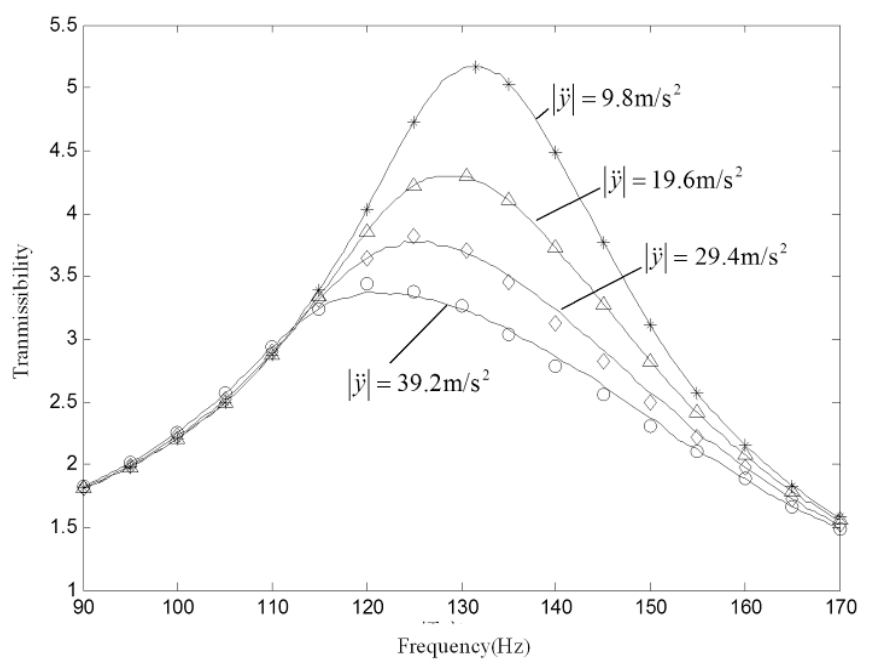

Fig. (5). Comparison of simulated vibration transmissibility curves with the experimental data (mass load is $10 \mathrm{~kg}$ ).

\section{CONCLUSION}

The dynamic property of honeycomb paperboard under vibration condition are different from that under the shock condition. Comparing with the identified results in $[7,8]$, honeycomb paperboard has greater damping and stiffness properties under shock condition than those under vibration condition, and the properties under shock condition are more sensitive to the load than those under vibration condition. Therefore, it is necessary to model the dynamic properties of honeycomb paperboard under shock and vibration conditions respectively.

Honeycomb paperboard has different vibration properties under different excitation conditions. The experimental results indicate that as the excitation amplitude increases, the stiffness decreases and the damping increases, meanwhile, with the increase of excitation force amplitude, the resonance frequency shifts to the left and the resonance peak decreases. Therefore, we can assume both the damping and stiffness properties are nonlinear. According to the recorded hysteresis loops at $120 \mathrm{~Hz}$, the elastic force in honeycomb paperboard is modeled as the polynomial of $z$ with only odd terms, the damping property is modeled as the combination of linear viscous damping and the quadratic type nonlinear damping.

A parameters identification process is formulated based on harmonic balance method, the parameters of the mass loaded honeycomb paperboard system under different load conditions are identified. The comparison of the simulated transmissibility curves with the experimental transmissibility 
indicate that the model is accurate, it can be used to simulate the $T_{r}-f$ curves of honeycomb paperboard under different load conditions.

\section{CONFLICT OF INTEREST}

The authors confirm that this article content has no conflict of interest.

\section{ACKNOWLEDGEMENTS}

This work is supported by natural fund of $\mathrm{GanSu}$ province (1212RJZA044, 1208RJZA147), The Young Scholars Science Foundation of Lanzhou Jiaotong University ( 2012039), and Lanzhou City Science Pillar Program(20134-107).

\section{REFERENCES}

[1] Y.F. Guo, and J.H. Zhang, "Shock absorbing characteristics and vibration transmissibility of honeycomb paperboard", Shock and Vibration, vol. 11, pp. 521-531, 2004.

[2] Y.F. Guo, "Experimental study of dynamic cushioning property and vibration transmissibility of honeycomb paperboard", PH. D. Thesis, Xi'an Jiaotong University, X'an, China, 2003 (in Chinese).

[3] D.M. Wang and Z.W. Wang, "Experimental investigation into the cushioning properties of honeycomb paperboard", Packaging Technology and Science, vol. 21, pp. 309-316, 2008.
[4] D.M. Wang, "Impact behavior and energy absorption of paper honeycomb sandwich panels", International Journal of Impact Engineering, vol. 36, pp.110-114, 2009.

[5] H. Kobayashi, and M. Daimaruya, "Dynamic and static compression tests for paper honeycomb cores and absorbed energy", JSME International Journal, vol. 41, pp. 338-344, 1998.

[6] L.X. Lu, Y.P. Sun, and Z.W. Wang, "Critical buckling load of paper honeycomb under out-of-plane pressure", Packaging Technology and Science, vol. 18, pp.141-150, 2005.

[7] D.P. Zhu, S.S. Zhou, and Z.K. Zhang, "Dynamic properties modeling and parameter identification for honeycomb fiberboard", Journal of Vibration and Shock, vol. 29, pp. 213217, 2010.

[8] D.P. Zhu, S.S. Zhou, and Z.K. Zhang, "Modeling and parameters identification of dynamic properties of paper honeycomb panel", in 2008 International Conference on Computer Science and Software Engineering, 2008, pp.417-420.

[9] J.S. Bendat, and A.G. Piersol, Single-Input/Output Relationships, Random Data-Analysis and measurement Procedures, Fourth Edition, New York, Wiley, 2010.

[10] A.H. Nayfeh and D.T. Mook, Forced Oscillations of Systems Having Single Degree of Freedom, Nonlinear Oscillations, Fourth Edition, New York, Wiley, 2004

[11] D.W. Jordan, and P. Smith, Periodic Solutions and Averaging Methods, Nonlinear Ordinary Differential Equations, Third Edition, Oxford, UK, Oxford University Press, 1998

[12] B.C. Wen, and Y.N. Li, System Linearization and Harmonic Balance Method, Nonlinear Oscillations in Engineering, Beijing, China, Science Press, 2007 (in Chinese).

[13] Z.H. Ni, Forced Vibration of Single Degree of Freedom system, Vibration Mechanics, Xi'an, China, Xi'an Jiaotong University Press, 1988. (in Chinese).

(c) Gao et al.; Licensee Bentham Open.

This is an open access article licensed under the terms of the Creative Commons Attribution Non-Commercial License (http://creativecommons.org/licenses/ by-nc/3.0/) which permits unrestricted, non-commercial use, distribution and reproduction in any medium, provided the work is properly cited. 